\title{
Effect of somatization disorder on social and work place functioning among upper socio-economic class adults attending outpatient clinic in national hospital, Abuja, Nigeria
}

Felix Ogbonnaya Iheme', Godswill Amechi Nnaji ${ }^{*}$, Lawrence Akinwale Moses ${ }^{1}$ and Olugbenga Ogunfowokan ${ }^{1}$

*Correspondence: godswilln@yahoo.co.uk

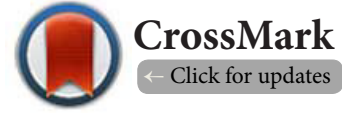

'Department of Family Medicine, National Hospital, Abuja, Nigeria.

${ }^{2}$ Department of Family Medicine, Nnamdi Azikiwe University Teaching Hospital, Nnewi, Anambra State, Nigeria.

\begin{abstract}
Background: Despite the common presentations of somatization in outpatient clinics, there is still a lot to be learnt on the subject, especially with regards to the various socioeconomic classes.

Aim of the study: To determine the role of somatization disorder on social and workplace functioning among patients in upper socioeconomic class in primary care setting.

Materials and methods: A cross sectional design with systematic random sampling technique was used. One hundred ninety five participants were studied from the upper socioeconomic class. A two part self-administered questionnaire was used. The ICD-10 criteria was incorporated into Patient Health Questionnaire (PHQ-15) and used in diagnosis of somatization disorder. Work place and social functioning assessment scale (WSAS) formed part of the second part of the questionnaire. SPSS version 16 was used for data analysis.
\end{abstract}

Results: The prevalence of somatization disorder in the study participants was $5.6 \%$. The impairment of functioning among participants with somatization disorder was higher in the following activities; ability to work $(\mathrm{OR}=1.13)$; to manage home $(\mathrm{OR}=1.24)$; to engage in private leisure $(\mathrm{OR}=1.56)$; and ability of participants to form and maintain close relationships with others $(\mathrm{OR}=1.53)$. In contrast, somatization disorder and the ability to engage in social leisure activities $(\mathrm{OR}=0.47)$ was negative or favourable.

Conclusion: Somatization disorder among the upper socioeconomic class is a risk factor for ability to work, manage homes, carry out private leisure activities, form and maintain close relationship with others, while it enhances ability for social leisure activities.

Keywords: Somatization disorder, somatoform disorder, social leisure activities, private leisure activities

\section{Introduction}

Somatization disorder is part of the Somatoform disorders complex (or Briquette's Syndrome) [1], which is a manifestation of stressdisorder characterized by physical symptoms that mimic physical injuries or disease with no identifiable physical cause [1]. The physical symptoms have no physiological explanation resulting in a large number of visits to doctors with unsatisfactory physical examinations and laboratory investigations [2]. The symptoms may persist for many years resulting in the patients being dissatisfied with their physicians. Others patients seek spiritual intervention in their illness, blaming their symptoms on some past sins or spiritual attack
[1]. Most times, a careful approach by a discerning physician would unravel the problem to the benefits of the patients [2].

This study is focused on individuals in the upper socioeconomic class because of the peculiarities of this class and the high incidence of stressful conditions seen here [3]. These include high incidence of sedentary lifestyle among them, high level policies and decisions they make regularly, their penchance to work under pressure in order to achieve targets and their personality orientation (type A personality group is achievement-oriented and are usually under pressure to deliver). In addition, the public is yet to develop any coping mechanism. The upper socioeconomic class include professionals who earn 
very well with a high job satisfaction in contrast with those in the middle class (semi-professionals), and the lower class (less well paid jobs and unemployed) $[4,5]$.

The impact of stress and its related disorders and socioeconomic effects are felt by the individuals (health and socioeconomic), the families, communities and even the individuals' work-places [6-8].

The individual may present with a variety of associated psychosomatic disorder, which may not be obvious to a nonsuspecting physician. These may range from depression, suicidal tendencies, anxiety neurosis, somatization disorders, substance abuse, peptic ulcers, hypertension and erectile dysfunctions, which may present as infertility [9].

In the work place, stress related disorders impact negatively on the quality of service delivery, which may result in increased loss of man-hours due to absenteeism, avoidable accidents, lack of motivation with poor commitment of staff to organisation's vision $[6,8]$.

Inspite of the fact that somatization disorders present commonly in the outpatient clinics, there is still a lot to be known on the subject, especially with regards to its prevalence in the various socioeconomic classes. Given the dearth of mental health specialists in most countries, care for somatization disorder is provided mainly by primary care providers [10]. Consequently, a large proportion of patients with psychological disorders do not receive appropriate treatment [8]. It has been observed that most patients seeking care for certain psychiatric disorders are more concerned about their physical than their psychological symptoms $[11,12]$. Prescription of nonspecific medications like hypnotics and vitamins by primary health care workers and private general practitioners in the developing nations for somatic complaints does not seem to help the situation $[13,14]$.

Dearth of data on the outcome of treatment trials in the developing countries has persisted even in the light of the findings on the public health importance of mental disorders [10].

\section{Aim and objectives}

To determine the prevalence of somatization disorder and its effect on social and workplace functioning among adult patients in upper socioeconomic class attending the general outpatient clinic at the National Hospital Abuja.

\section{Materials and methods Study setting}

The study was conducted in the General Outpatient Department (GOPD) of the National Hospital, Federal capital city, Abuja, Nigeria. The Federal Capital Territory (FTC) comprises six area councils- the Abuja municipal area council (AMAC), Bwari, Kuje, Kwali, Abaji and Gwagwalada area councils. FCT has population of about 1.4 million by 2006 [15]. The National Hospital, Abuja (NHA) is a 300-bedded health facility that provides primary, secondary and tertiary medical care to residents of FCT and its neighbouring states (Nassarawa, Niger, Benue, Kaduna, Bauchi, Gombe, Taraba and Adamawa) as referrals from across the nation and even beyond. The GOPD in NHA serves as a primary care facility manned by consultant family physicians, resident doctors in family medicine, medical officers and National youth service doctors. The general outpatient clinic and the general pediatric outpatient clinic in the department of Family Medicine are the points of entry of most patients into the hospital. About 64,104 patients were seen in both clinics in 2008 with a monthly average of 5,342. This number, included 4,113 (77\%) adults of every social strata, and 1,229 (23\%) children. Patients with somatization disorders constituted 2,188 adults (average of 182 monthly) seen and treated in 2008.

\section{Study design}

This was a descriptive cross-sectional study designed to ascertain the proportion of patients of the upper socioeconomic class who presented with somatization disorder and impairment of abilities for social, private leisure and work activities.

\section{Population of the study}

The population of the study include patients in the upper socioeconomic class who attended the general out-patient clinic of the hospital during the study period (July to September, 2010).

\section{Sample method}

A systematic random sampling technique was used in the study. A list of eligible adult patients attending the general outpatient clinic, who belong to the upper socioeconomic class was prepared on a daily basis. The first name to be selected on this list was done by balloting of the first three names on the list. Subsequently, every third consecutive patient on the list was selected for enrolment in the study until the desired sample size was attained.

\section{Inclusion criteria}

Adult patients aged 18 years and above belonging to upper socioeconomic class who presented with the following; four symptoms of pain, two symptoms in the digestive tract, one symptom involving the sexual organs, and one symptom related to the nervous system with duration of two years and above and not accounted for by medical illness [16].

\section{Exclusion criteria}

Included those patients with organic illness, those diagnosed with a psychiatric illness and patients who were already on treatment for somatization disorder.

\section{Sample size}

Thesamplesizedetermination was determinedusing theVaughan formula; 


$$
\mathrm{n}=\frac{\mathrm{z}^{2} \mathrm{pq}}{\mathrm{d}^{2}}
$$

$z=$ the standard normal deviate, usually set at $1.96,(95 \%$ confidence level).

$\mathrm{p}=$ the proportion of the target population estimated to have a somatization disorder (or prevalence) which was 12.4 $[17,18]$.

$\mathrm{q}=1.0-\mathrm{p}$. and $\mathrm{d}=$ degree of accuracy desired set at 0.05

$$
\mathrm{n}=\frac{(1.96)^{2} \times 0.124 \times 0.876}{0.0025} \quad \mathrm{n}=167
$$

Adjusting for population less than $10,000=\mathrm{nf}$ (desired sample size).

$$
n f=\frac{n}{1-\frac{(n)}{(N)}}
$$

Where $\mathrm{nf}=$ the desired sample size when population is less than 10,000

$\mathrm{n}=$ the desired sample size when population is more than 10,000

$\mathrm{N}=$ the estimate of population size $(3,600)$

The desired sample size $(\mathrm{n})$ when population is more than 10,000 is 167 .

Therefore

$$
\begin{gathered}
\mathrm{nf}=\frac{\frac{167}{1-\frac{(167)}{(3600)}}}{=\frac{167}{1-0.046}=175.125 \text { (Minimum sample size) }}
\end{gathered}
$$

Using attrition rate of $10 \%,=75.125 \times 1.01=193$;

Therefore minimum Sample size, $\mathrm{nf}=193$

\section{Ethical approval}

Ethical approval for the study was obtained from the ethics committee of the National Hospital, Abuja. Eligible patients were recruited after informed, written consent was obtained from them.

\section{Instruments for data collection}

Well-structured, two part self-administered questionnaire was used. The ICD-10 criteria was incorporated into Patient Health Questionnaire (PHQ-15) and used in diagnosis of somatization disorder. Work place and social functioning assessment scale (WSAS) formed part of the second part of the questionnaire. Also, Weighing scales, Stadiometer, Digital thermometer, Sphygmomanometer, and the ICD 10 disease classification were used as instrument for data collection.

\section{Study protocol}

The recruitment of the patients was undertaken between the months of July and September 2010 at the general outpatient clinic of the National Hospital, Abuja. This was done with the support of a trained assistant and the nurses on duty. At first, an explanation of the protocol was made at the general out-patient as they waited to see the doctors. An adapted socioeconomic screening tool modified by Ogunlesi et al., for use in developing countries was adopted as criteria for selecting eligible list of patients in upper socioeconomic class [5]. Thereafter, systematic sampling method was used to select patients from the list. The patients who accepted to participate in the study were made to sign or thumb print a written informed consent form.

Thereafter, a pretested two-part questionnaire was administered on the selected patients. The first part of the questionnaire elicited the participants' socio-demographic data, including age, gender, religion, ethnic group, marital status and place of abode. Questions were also asked regarding medical and drug histories and alcohol ingestion. The second part of the questionnaire incorporated items from both the ICD 10 criteria for diagnosis of somatization disorder and the 13-item Patients Health Questionnaire (Prime MD scale 15) component assessing for somatization disorder. The total number of positive responses to the items in the ICD 10 and PHQ-15 component criteria was calculated. The participants were also taken through a symptom checklist.

A general physical examination was then performed on each participant, by the investigators. The height and weight were measured using a stadiometer attached to the weighing balance (ZT-160 Health Scale, United Kingdom) without shoes or headgear. The weight measurement was standardized by using the same scale for all the patients, and recalibration to zero before weighing of each patient. The body mass index (BMI) was calculated as the quotient of the weight in kilograms, and the height in meters squared $\left(\mathrm{kg} / \mathrm{m}^{2}\right)$. A clinical assessment for pallor, jaundice, and pedal oedema was then undertaken. The axillary temperature was measured, using a digital thermometer. The radial pulse was then counted for a minute, and the blood pressure was measured with the patient in sitting position, using mercury in glass sphygmomanometer (Mercurex-Spengler, France) with an adult-size cuff $(12 \times 27 \mathrm{~cm}$ and $16 \times 32 \mathrm{~cm})$. The neck was also examined for any evidence of thyroid enlargement, while the hands were examined for tremors and sweating.

Definition of terms: Somatization disorder (also Briquet's syndrome or hysteria) is a somatoform disorder characterized by recurring, multiple, and current, clinically significant complaints about somatic symptoms. Symptoms often include reports of pain, gastrointestinal distress, sexual problems, and pseudo neurological symptoms [1].

Somatization disorder can be diagnosed in patients who presented with three out of the 13-item PHQ-15 criteria updated with the ICD-10 criteria, which specified a total duration of at least 2 years of the symptoms. PHQ-15 is a self-administered questionnaire, which is useful in screening people for somatization disorder and for monitoring somatic symptom severity in both clinical practice and research [19].

Home management refers to tidying, cooking, shopping, cleaning, and looking after the children and the home, paying bills, etc. 
Iheme et al. Family Practice Reports 2014,

Social leisure activities with other people include parties, bars, outings, clubs, visits, dating, home entertainment, etc.

Private leisure activities done alone include gardening, collecting, sewing and walking alone, etc.

\section{Statistical analysis}

Data analysis was done with Statistical package for the Social Sciences (SPSS) software version 16 . The association between somatization disorder and dependent variables were tested using Odds Ratio tests for categorical variables. A p value of 0.05 or less was considered to be significant in all analyses.

\section{Results}

The mean age of the participants was 37 years with 30 to 39 years group being the most frequent age group, $44.1 \%$. Female participants were a majority, $74.4 \%$ (145 of 195), while those married were in majority, $82.6 \%$ (161 of 195). Most of the participants, $85.1 \%$ (166 of 195) were city dwellers, while 14.9\% (29 of 195) were Satellite town dwellers (Table 1).

Majority of the participants, $82.6 \%$ (161 of 195) had tertiary education. A high proportion of the participants, $44.6 \%$ (87) were employed in the public sector compared with those in business or self-employment, $31.8 \%$ (62) and $23.6 \%$ (46), respectively. A majority $71.8 \%$ (140 of 195) of the participants was Christians.

Participants of Igbo tribe constituted $30.8 \%(60)$, while other Nigerian tribes were $34.3 \%$ (67). Nuclear families accounted for $54.9 \%$ (107 of 195), while $30.3 \%$ (59) were of extended families. The type of marriage was mainly monogamy, $82.1 \%$ (160 of 195), while polygamy accounted for $15.4 \%$ (30 of 195) (Table 2).

\section{Discussion}

Overview

The results of this study on somatization disorder showed significant findings among the upper socioeconomic class patients presenting with multiple non-specific somatic complaints at the General Outpatient clinic of National Hospital, Abuja.

\section{Prevalence of somatization disorder}

A small proportion of participants in this study (5.6\%) who presented with multiple somatic symptoms met the ICD 10 criteria for somatization disorder [21].

This result is comparable to various studies between somatic symptoms and somatization disorder, which reported that an average of $2.7 \%$ to $9.4 \%$ of patients who presented with somatic symptoms had somatization disorder. These studies were however carried out in the general populations and not limited to any socioeconomic class. Furthermore, more questionnaires including the Whiteley-7, the Diagnostic interview Schedule (DIS), the Somatic Symptom index, and a modified version of the Whiteley index (WI), were all employed in their studies. In Nigeria, Gureje et al., observed a prevalence of $1.1 \%$ for somatization disorder, while the figure by Ohaeri et al., was
8.4\% for probable somatoform disorders in Nigeria $[22, \mathbf{2 3}]$. The reason for the difference in the rate is probably due to

Table 1. Socio-demographic characteristics of the study subjects.

\begin{tabular}{|c|c|c|}
\hline $\begin{array}{l}\text { Socio-demographic } \\
\text { Characteristic }\end{array}$ & $\begin{array}{l}\text { Frequency } \\
(\mathrm{N}=195)\end{array}$ & $\begin{array}{l}\text { Percentage } \\
(\%)\end{array}$ \\
\hline \multicolumn{3}{|l|}{ 1. Age Range (Years) } \\
\hline$\leq 20$ & 0 & 0 \\
\hline$>20-\leq 30$ & 30.0 & 15.4 \\
\hline$<30-\leq 40$ & 86.0 & 44.1 \\
\hline$<40-\leq 50$ & 49.0 & 25.1 \\
\hline$<50-\leq 60$ & 5.0 & 2.6 \\
\hline \multicolumn{3}{|l|}{ 2. Sex } \\
\hline Male & 50.0 & 25.6 \\
\hline Female & 145.0 & 74.4 \\
\hline \multicolumn{3}{|l|}{ 3. Marital status } \\
\hline Single & 16.0 & 8.2 \\
\hline Married & 161.0 & 82.6 \\
\hline Divorced & 13.0 & 6.7 \\
\hline Separated & 2.0 & 1.0 \\
\hline Widowed & 3.0 & 1.5 \\
\hline \multicolumn{3}{|l|}{ 4. Dwelling Place } \\
\hline City centre & 166.0 & 85.1 \\
\hline Satellite Town & 29.0 & 14.9 \\
\hline \multicolumn{3}{|l|}{ 5. Educational Status } \\
\hline None & 8.0 & 4.1 \\
\hline Primary & 4.0 & 2.0 \\
\hline Secondary & 22.0 & 11.3 \\
\hline Tertiary & 161.0 & 82.6 \\
\hline \multicolumn{3}{|l|}{ 6. Occupation } \\
\hline Public Service & 87.0 & 44.6 \\
\hline Businessman/Self Employed & 62.0 & 31.8 \\
\hline Employed (Private) & 46.0 & 23.6 \\
\hline \multicolumn{3}{|l|}{ 7. Religion } \\
\hline Christianity & 140.0 & 71.8 \\
\hline Islam & 49.0 & 25.1 \\
\hline Traditional & 2.0 & 1.0 \\
\hline None & 4.0 & 2.1 \\
\hline \multicolumn{3}{|l|}{ 8. Tribe } \\
\hline Yoruba & 39.0 & 20.0 \\
\hline Ibo & 60.0 & 30.8 \\
\hline Hausa & 29.0 & 14.9 \\
\hline Others & 67.0 & 34.3 \\
\hline \multicolumn{3}{|l|}{ 9. Type of Family } \\
\hline Single Parent & 20.0 & 10.2 \\
\hline Nuclear & 107.0 & 54.9 \\
\hline Extended & 59.0 & 30.3 \\
\hline Others & 9.0 & 4.6 \\
\hline \multicolumn{3}{|l|}{ 10. Type of Marriage } \\
\hline Monogamous & 160.0 & 82.1 \\
\hline Polygamous & 30.0 & 15.4 \\
\hline Same Sex & 5.0 & 2.5 \\
\hline
\end{tabular}


Iheme et al. Family Practice Reports 2014,

Table 2. Association between Individual's Workplace and Social Functioning (using the Modified Workplace and Social Assessment Scale [WSAS]) and Somatization Disorder [20].

\begin{tabular}{|c|c|c|c|c|c|}
\hline & & \multicolumn{2}{|c|}{$\begin{array}{l}\text { Somatization } \\
\text { Disorder }\end{array}$} & \multirow{2}{*}{$\begin{array}{l}\text { Total } \\
-- \\
\end{array}$} & \multirow{2}{*}{$\begin{array}{l}\text { Odds } \\
\text { Ratio } \\
-- \\
\end{array}$} \\
\hline & & Yes & No & & \\
\hline \multirow[t]{3}{*}{ Impaired Ability to Work } & Yes & 4 & 59 & 63 & 1.13 \\
\hline & No & 7 & 125 & 132 & -- \\
\hline & Total & 11 & 184 & 195 & -- \\
\hline \multirow[t]{3}{*}{ Impaired Home Management } & Yes & 5 & 68 & 73 & 1.24 \\
\hline & No & 6 & 116 & 122 & -- \\
\hline & Total & 11 & 184 & 195 & -- \\
\hline \multirow[t]{3}{*}{ Impaired Social Leisure } & Yes & 5 & 180 & 185 & 0.47 \\
\hline & No & 6 & 4 & 10 & -- \\
\hline & Total & 11 & 184 & 195 & -- \\
\hline \multirow[t]{3}{*}{ Impaired Private Leisure } & Yes & 7 & 76 & 83 & 1.56 \\
\hline & No & 4 & 108 & 112 & -- \\
\hline & Total & 11 & 184 & 195 & -- \\
\hline \multirow{3}{*}{$\begin{array}{l}\text { Ability to Form And } \\
\text { Maintain Relationship }\end{array}$} & Yes & 5 & 56 & 61 & 1.53 \\
\hline & No & 6 & 128 & 134 & -- \\
\hline & Total & 11 & 184 & 195 & -- \\
\hline
\end{tabular}

The two by two contingency table was formed by merging the responses of all four grades of impairment in each category into Yes and No. Table 2 above depicts that somatization disorder is associated with the Impaired Ability to Work, (OR 1.13) Impaired Home Management (OR 1.24), Impaired Private Leisure activities (OR 1.56) and Ability to form and maintain relationship (OR 1.53); however there is no association between somatization disorder and Impaired Social Leisure activities (OR 0.47) in this study.

the fact that all these studies were non-discriminatory with respect to socioeconomic class unlike in this study.

Direct comparison of prevalence studies for somatization disorders is difficult, due to a lack of homogeneity [24]. Studies differ in terms of culture, patient population, socio-demographic factors, diagnostic instruments, and methodology [22]. Furthermore, some studies focus on somatoform disorders alone, while others include all forms of mental illness $[\mathbf{2 5}, \mathbf{2 6}$.

The symptom list in the above studies was the same as the list of physical complaints of DSM-III-R somatization disorder. Prevalence of between 0.1-0.8 percent for full somatization disorder have been reported in studies done in South Africa, Germany and the USA $[17,27,28]$.

Most detection of somatization disorder fall below the range of $50 \%$ in primary care when DSM-IV criteria is used [29]. The rates are thought to be even lower in Africa [24]. A combination of somatic presentation, stigmatization of mental illness, limited physician time and training may all contribute to this shortcoming $[25,30]$. It has been stated variously that the reporting of somatic symptoms by patients may not mean a willingness to recognize mental distress but a belief that reporting may be an easier route for seeking help from the family physician, and may thus serve as a means of entry to the primary care facility. Therefore, somatization and other psychological disorders may not be recognized if not looked out for.

This study found statistically significant association between manifestation of somatization disorder and effects on certain observed parameters both in the workplace and in the society using the workplace and social functioning assessment scale (WSAS).

\section{Somatization disorder and the workplace and social functioning}

The finding in this study of a statistically significant association between impaired ability of the participants to work and somatization disorder is biologically plausible. Somatization disorder, though, has no physical basis, can lead to poor performance of tasks by workers in their workplaces. This finding is corroborated with the findings of other authors in other locations [8,31-34]. The implication of these findings is that people with somatization disorder would not be able to function properly in their workplace as a result of loss of manpower, poor output, avoidable workplace accidents, loss of jobs as well as further impact on finances, family and psychosocial wellbeing. Consequently, somatization disorder can lead to a reduction in productivity of the worker and ulti-mately socioeconomic downturn of the nation as a whole.

Similarly, the association of somatization disorder with increased impairment of private leisure (activities done alone, such as reading, gardening, sewing, etc) is probably due to the tendency to avoid activities that require mental concentration by the patients. It is probable that as somatization disorder increased individuals moved from private leisure activities to social leisure activities with others, such as attending parties, bars, outing, clubs, dating and home entertainment $[6,8]$.

Secondly, the finding of this study show that somatization disorder would lead to a reduction in the impairment of social leisure activities, i.e., enhancement of social leisure activities; thus those with somatization disorder would tend to engage more in social leisure activities such as attending parties, bars, clubs, dating, visiting, home entertainment, etc. This is probably a coping strategy, whereby going out to bars and parties could provide the opportunity to drink, socialize and reduce psychosocial stress levels, while enhancing relaxation. Ultimately, the reduction in the impairment of social leisure could lead to effective or ineffective coping mechanisms which could result in the appropriate use of recreational activities with obvious positive influence as compared with negative coping mechanisms evidenced by alcohol use. This is inherent in the behavioral disposition of stressed-up workers such as coping strategies as a form of adaptation. The causes of stress in the work place include: the demands inherent in different tasks, job characteristics, instability and demand, role, workplace culture, interpersonal, and family [32]. Workplace and social stress impact negatively on both the individual and the family, as well as, on the society and therefore calls for concerted efforts by all [34]. 
Iheme et al. Family Practice Reports 2014,

Similarly, there is an association between somatization disorder and ability to form and maintain close relationship with others including those living with the patient.

It implies that somatization disorder probably affects the interpersonal relationship probably by causing people to limit their association with others resulting in being withdrawn, while feeling ill. The culture of the people determines acceptable illness behavior, however, most cultures allow for reduced activities and social protection of the ill person during the period of convalescence. In this case, somatization being a chronic problem, may result in social withdrawal of the individual involved. This partly explains why severely impaired social leisure activities with others are linked with very low proportion of somatization disorder. People who are stressup may want to relieve this by finding ways and means of distracting their minds away from the somatization problem.

Also, the impairment of ability to manage home is greater within somatization disorder group. The tasks involved in home management include the following; tidying, cooking, shopping, cleaning, children upbringing, and payment of bills, etc. The inability of the patients to discharge these tasks is probably related to poor mental concentration and perceived physical incapacitation by such patients. The implication is that the immediate members of the family of the patient would feel the impact of poor home management, which on the larger scale may lead to family dysfunction with its attendant problems.

It is probable that some independent variables in the workplace, may in association with other remote factors increase the individual level of stress and thus the likelihood of manifestation of somatization disorder.

\section{Conclusion}

There is a high prevalence of somatization disorder among the upper socioeconomic class, among the attendees at the outpatient clinic which has a direct effect on ability to work, management of home, private leisure activities, and ability to form and maintain close relationship with others. In contrast, there is an inverse relationship between somatization disorder and social leisure activities.

\section{Recommendation}

Increasing the diagnostic and therapeutic capabilities of primary care physicians in the diagnosis of somatization disorder by conducting regular updates on mental health. The link between somatization disorder and alcohol abuse needs to be studied. There is need for further studies to be carried out on the effect of family functioning and family types on somatization disorder.

\section{Limitation of the study}

Theoretically, there is probability of some errors occurring during classification of participants since the researchers relied on information provided by participants. Also, there could be error in recall of somatic symptoms.

Competing interests

The authors declare that they have no competing interests.

Authors' contributions

\begin{tabular}{|l|c|c|c|c|}
\hline Authors' contributions & FOI & GAN & LAM & OO \\
\hline Research concept and design & $\checkmark$ & - & $\checkmark$ & $\checkmark$ \\
\hline Collection and/or assembly of data & $\checkmark$ & -- & -- & -- \\
\hline Data analysis and interpretation & $\checkmark$ & $\checkmark$ & $\checkmark$ & $\checkmark$ \\
\hline Writing the article & $\checkmark$ & $\checkmark$ & $\checkmark$ & $\checkmark$ \\
\hline Critical revision of the article & -- & $\checkmark$ & -- & -- \\
\hline Final approval of article & -- & $\checkmark$ & -- & -- \\
\hline Statistical analysis & $\checkmark$ & $\checkmark$ & -- & -- \\
\hline
\end{tabular}

Acknowledgement

The authors acknowledge the contribution of nurses and other staff of the Department of Family Medicine, National Hospital, Abuja, Nigeria in making this work a success.

\section{Publication history}

Editor: Radha Y. Aras, Yenepoya University, India.

Received: 01-May-2014 Final Revised: 03-Jul-2014

Accepted: 09-Jul-2014 Published: 16-Jul-2014

\section{References}

1. Somatoform disorders. 2010.

2. Somatoform disorders. Wrong diagnosis. 2010. | Website

3. Cohen S, Doyle WJ and Baum A. Socioeconomic status is associated with stress hormones. Psychosom Med. 2006; 68:414-20. | Article I PubMed

4. William T and Hickey J. Society in Focus: Boston, MA: Pearson; 2005: 56-56. | Pdf

5. Ogunlesi TA, Dedeke IOF and Kuponiyi OT. Socioeconomic Classification of school children attending specialist paediatric centres in Ogun State, Nigeria. The Nigeria medical Practitioner. 2008; 54:21-25. | Article

6. Work health, Reducing occupations stress. 2009. I Website

7. Price S.J. Families and Change. Coping with Stress events and transitions. $4^{\text {th }}$ Edition. Minnesota: Sage publishers. 2014; 90-120.

8. Stanic J. Productivity drops, workplace stress increases. Dynamic business. 2009. I Website

9. Smith M. Understanding Stress. Help guide. 2008. | Website

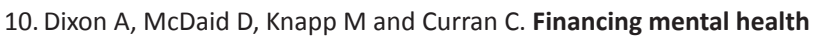
services in low- and middle-income countries. Health Policy Plan. 2006; 21:171-82. | Article | PubMed

11. Demyttenaere K, Bruffaerts R, Posada-Villa J, Gasquet I, Kovess V, Lepine JP, Angermeyer MC, Bernert S, de Girolamo $G$ and Morosini P et al. Prevalence, severity, and unmet need for treatment of mental disorders in the World Health Organization World Mental Health Surveys. JAMA. 2004; 291:2581-90. | Article | PubMed

12. Sartorius N. Physical symptoms of depression as a public health concern. J Clin Psychiatry. 2003; 64 Suppl 7:3-4. | PubMed

13. Patel V, Chisholm D, Rabe-Hesketh S, Dias-Saxena F, Andrew $G$ and Mann A. Efficacy and cost-effectiveness of drug and psychological treatments for common mental disorders in general health care in Goa, India: a randomised, controlled trial. Lancet. 2003; 361:33-9. | Article | PubMed

14. Quan $M$ and Gitlin M. Recognition and management of depression. Monograph sponsored by the California Academy of Family Physicians. 2004.

15. Federal Capital Territory (Nigeria), Wikipedia. 2011. 
Iheme et al. Family Practice Reports 2014,

16. Medical-dictionary. 2014. | Website

17. Dickinson WP, Dickinson LM, deGruy FV, Candib LM, Main DS, Libby $\mathrm{AM}$ and Rost $\mathrm{K}$. The somatization in primary care study: a tale of three diagnoses. Gen Hosp Psychiatry. 2003; 25:1-7. I Article I PubMed

18. Bener A, Ghuloum S, Al-Mulla AA, Al-Marri S, Hashim MS and Elbagi IE. Prevalence of somatisation and psychologisation among patients visiting primary health care centres in the State of Qatar. Libyan J Med. 2010; 5:1-7. | Article | PubMed Abstract | PubMed Full Text

19. Kroenke K, Spitzer RL and Williams JB. The PHQ-15: validity of a new measure for evaluating the severity of somatic symptoms. Psychosom Med. 2002; 64:258-66. | Article | PubMed

20. Kanchanaraksa S. Estimating Risk, John Hopkins Bloomberg School of Public Health. 2014. | Pdf

21. International statistical classification of diseases and related health problems (ICD-10). Tenth revision. Canadian Institute for Health Information. 2006; 1:189-256. | Book

22. Escobar JI and Gureje O. Influence of cultural and social factors on the epidemiology of idiopathic somatic complaints and syndromes. Psychosom Med. 2007; 69:841-5. I Article I PubMed

23. Ohaeri JU and Odejide OA. Somatization symptoms among patients using primary health care facilities in a rural community in Nigeria. Am J Psychiatry. 1994; 151:728-31. I Article I PubMed

24. The World health Report. Mental Health: New understanding, New Hope. World Health Organization. 2001. | Website

25. Omar MA, Green AT, Bird PK, Mirzoev T, Flisher AJ, Kigozi F, Lund C, Mwanza J and Ofori-Atta AL. Mental health policy process: a comparative study of Ghana, South Africa, Uganda and Zambia. Int J Ment Health Syst. 2010; 4:24. | Article I PubMed Abstract | PubMed Full $\underline{\text { Text }}$

26. Malomo IO, Aina OF, Ladapo HT and Owoeye AO. Ten-year mortality review in a pioneer psychiatric hospital in West Africa. East Afr Med J. 2003; 80:379-83. | PubMed

27. Lieb R, Zimmermann P, Friis RH, Hofler M, Tholen S and Wittchen HU. The natural course of DSM-IV somatoform disorders and syndromes among adolescents and young adults: a prospective-longitudinal community study. Eur Psychiatry. 2002; 17:321-31. I Article I PubMed

28. Ladwig KH, Marten-Mittag B, Erazo N and Gundel H. Identifying somatization disorder in a population-based health examination survey: psychosocial burden and gender differences. Psychosomatics. 2001; 42:511-8. | Article | PubMed

29. Becker SM. Detection of somatization and depression in primary care in Saudi Arabia. Soc Psychiatry Psychiatr Epidemiol. 2004; 39:962-6. | Article I PubMed

30. Patel V and Kleinman A. Poverty and common mental disorders in developing countries. Bull World Health Organ. 2003; 81:609-15. | Article | PubMed Abstract | PubMed Full Text

31. Wang J, Smailes E, Sareen J, Fick GH, Schmitz N and Patten SB. The prevalence of mental disorders in the working population over the period of global economic crisis. Can J Psychiatry. 2010; 55:598-605. Article | PubMed

32. Ayala $E$ and Carnero AM. Determinants of burnout in acute and critical care military nursing personnel: a cross-sectional study from Peru. PLOS One. 2013; 8:e54408. | Article | PubMed Abstract | PubMed Full Text

33. Mojoyinola JK. Effects of Job Stress on Health, personal and Work Behaviour of Nurses in Public Hospitals in Ibadan metropolis, Nigeria. Ethno-Med. 2008; 2:143-148. I Pdf

34. Godin I and Kittel F. Differential economic stability and psychosocial stress at work: associations with psychosomatic complaints and absenteeism. Soc Sci Med. 2004; 58:1543-53. I Article I PubMed

\section{Citation:}

Iheme FO, Nnaji GA, Moses LA and Ogunfowokan O. Effect of somatization disorder on social and work place functioning among upper socio-economic class adults attending outpatient clinic in national hospital, Abuja, Nigeria. Fam Pract Rep. 2014; 1:1. http://dx.doi.org/10.7243/2056-5690-1-1 